\title{
MODELING A CORONAL LOOP HEATED BY MAGNETOHYDRODYNAMIC TURBULENCE NANOFLARES
}

\author{
F. Reale, ${ }^{1}$ G. Nigro, ${ }^{2}$ F. Malara, ${ }^{2}$ G. Peres, ${ }^{1}$ and P. Veltri ${ }^{2}$ \\ Received 2005 January 7; accepted 2005 June 27
}

\begin{abstract}
We model the hydrodynamic evolution of the plasma confined in a coronal loop, 30,000 $\mathrm{km}$ long, subject to the heating of nanoflares due to intermittent magnetic dissipative events in the MHD turbulence produced by loop footpoint motions. We use the time-dependent distribution of energy dissipation along the loop obtained from a hybrid shell model, occurring for a magnetic field of about $10 \mathrm{G}$ in the corona; the relevant heating per unit volume along the loop is used in the Palermo-Harvard loop plasma hydrodynamic model. We describe the results, focusing on the effects produced by the most intense heat pulses, which lead to loop temperatures between 1 and $1.5 \mathrm{MK}$.
\end{abstract}

Subject headings: Sun: activity — Sun: corona

\section{INTRODUCTION}

Nanoflares (Parker 1988) are among the best candidates to explain the heating of the solar corona and, in particular, of the coronal loops (e.g., Peres et al. 1993; Cargill 1993; Kopp \& Poletto 1993; Shimizu 1995; Judge et al. 1998; Mitra-Kraev \& Benz 2001; Katsukawa \& Tsuneta 2001; Warren et al. 2002, 2003; Spadaro et al. 2003; Cargill \& Klimchuk 1997, 2004; Müller et al. 2004; Testa et al. 2005).

Although the evidence of nanoflares appears to be well established, it is still unclear whether, and to what extent, they really can provide enough energy to heat the whole corona (e.g., Aschwanden 1999). More recently, models of nanoflares with a prescribed random time distribution of the pulses deposited at the footpoints of multistranded loops have been proposed (Warren et al. 2002, 2003) and have been shown to describe several observed features.

According to some models, nanoflares are the result of dissipation in magnetohydrodynamic (MHD) turbulence, generated inside closed magnetic structures in the corona, and due to nonlinear interactions among fluctuations generated by photospheric motions. Possible evidence of turbulent motions has been detected from line broadenings in coronal loops (Saba \& Strong 1991). Most of these models include direct numerical solutions of MHD equations in two or three dimensions (Einaudi et al. 1996; Hendrix \& Van Hoven 1996; Dmitruk \& Gómez 1997, 1998, 1999; Buchlin et al. 2003) using relatively low Reynolds/Lundquist numbers. Recently, Nigro et al. (2004, hereafter NMCV04) have related coronal nanoflares to intermittent dissipative events in the MHD turbulence produced in a coronal magnetic structure by footpoint motions. The injected energy is stored in the loop up to significant levels in the form of magnetic and velocity fluctuations and released intermittently through nonlinear interactions that process these fluctuations and generate cascades toward smaller scales where energy is dissipated. The derived probability distribution functions of the peak maximum power, peak duration time, energy dissipated in a burst, and waiting time between bursts are in good agreement with those obtained from the analysis of coronal impulsive events (Datlowe et al. 1974; Lin et al. 1984; Dennis 1985; Crosby et al. 1993; Shimizu \& Tsuneta 1997; Krucker \&

\footnotetext{
1 Dipartimento di Scienze Fisiche ed Astronomiche, Sezione di Astronomia, Università di Palermo, Piazza del Parlamento 1, I-90134 Palermo, Italy.

2 Dipartimento di Fisica, Università della Calabria, I-87030 Arcavacata di Rende, Italy.
}

Benz 1998; Boffetta et al. 1999; Parnell \& Jupp 2000; Aschwanden et al. 2000a, 2000b). This heating model does not require any ad hoc hypothesis once the loop length and the characteristic Alfvén speed, i.e., the strength of the ambient magnetic field (if the density does not change much), are fixed.

Here we model the plasma confined in a coronal loop heated according to the event dissipation rate and distribution described in NMCV04. We compute the evolution of the distributions of the density, temperature, and velocity of the loop plasma by means of the time-dependent thermohydrodynamic Palermo-Harvard (Peres et al. 1982; Betta et al. 1997) loop model, assuming the output of the hybrid shell model illustrated in NMCV04 as the basis of the heating function.

In $\S 2$ we describe the setup of the loop model with the MHDturbulence dissipation rate as input heating. In $\S 3$ we show relevant results, and we discuss them in $\S 4$.

\section{THE LOOP MODEL}

Our purpose here is to model the evolution of the plasma confined in a coronal loop under the effect of the energy dissipation predicted in NMCV04. According to their settings, we model a magnetic loop with a total length of $30,000 \mathrm{~km}$. The plasma is described as a compressible fluid moving and transporting energy only along the magnetic field lines, i.e., along the loop itself. Thus, the magnetic field has only the role of confining the plasma. The loop model assumes a constant loop cross section.

We use the Palermo-Harvard code (Peres et al. 1982; Betta et al. 1997), a one-dimensional hydrodynamic code that consistently solves the time-dependent density, momentum, and energy equations for the plasma confined by the magnetic field:

$$
\begin{gathered}
\frac{d n}{d t}=-n \frac{\partial v}{\partial s} \\
n m_{\mathrm{H}} \frac{d v}{d t}=-\frac{\partial p}{\partial s}+n m_{\mathrm{H}} g+\frac{\partial}{\partial s}\left(\mu \frac{\partial v}{\partial s}\right) \\
\frac{d \epsilon}{d t}+(p+\epsilon) \frac{\partial v}{\partial s}=H-n^{2} \beta P(T)+\mu\left(\frac{\partial v}{\partial s}\right)^{2}+\frac{\partial}{\partial s}\left(\kappa T^{5 / 2} \frac{\partial T}{\partial s}\right)
\end{gathered}
$$

with $p$ and $\epsilon$ defined by

$$
p=(1+\beta) n K_{\mathrm{B}} T \epsilon=\frac{3}{2} p+n \beta \chi,
$$


where $n$ is the hydrogen number density, $s$ the spatial coordinate along the loop, $v$ the plasma velocity, $m_{\mathrm{H}}$ the mass of the hydrogen atom, $\mu$ the effective plasma viscosity, $P(T)$ the radiative losses function per unit emission measure, $\beta$ the fractional ionization, i.e., $n_{e} / n_{\mathrm{H}}, \kappa$ the thermal conductivity (Spitzer 1962), $K_{\mathrm{B}}$ the Boltzmann constant, and $\chi$ the hydrogen ionization potential; $H(s, t)$ is a function of both space and time, which describes the heat input in the loop. This function is described in detail in $\S 2.1$. The numerical code uses an adaptive spatial grid to adequately follow the evolving profiles of the physical quantities, which can vary dramatically in the transition region and under the effect of the evolution. The loop is not symmetric, the apex is at half the numerical grid, and there is a chromosphere on each side. The boundary conditions at the loop footpoints are the same as in Reale et al. (2000).

\subsection{The Heating Function}

The original version of the Palermo-Harvard hydrodynamic code includes a space- and time-dependent heating function, which describes the input of external energy triggering transient events (Peres et al. 1987). Several formulations are possible, and the code can be easily adapted. For this work, the heating function is given by the output dissipation rate of NMCV04 (in the form of a numerical table).

The model developed in NMCV04 has been derived within the framework of reduced magnetohydrodynamics (RMHD; Strauss 1976; Zank \& Matthaeus 1992) with the assumptions that (1) the plasma is permeated by a strong uniform magnetic field $\boldsymbol{B}_{0}$ in the longitudinal direction; (2) there is a low thermal-to-magnetic pressure ratio, $\beta_{P}=8 \pi p / B^{2} \ll 1$; (3) the longitudinal scale $l_{\|}$of transverse velocity $v_{\perp}$ and magnetic field $B_{\perp}$ fluctuations is much larger than the transverse scale $l_{\perp}$; indeed, the MHD turbulence is anisotropic (e.g., Carbone \& Veltri 1990), the energy cascade being more efficient perpendicularly to $\boldsymbol{B}_{0}$; and (4) small amplitude perturbations $B_{\perp} / B_{0}=v_{\perp} / c_{\mathrm{A} 0}<l_{\perp} / l_{\|} \ll 1$, where $c_{\mathrm{A} 0}$ is the background Alfvén velocity, commonly assumed to be of the order of $c_{\mathrm{A} 0} \sim 10^{8} \mathrm{~cm} \mathrm{~s}^{-1}$, while the fluctuating velocity can be estimated using nonthermal broadening of coronal spectral lines, $v_{\perp} \sim 3 \times 10^{6}-1.5 \times 10^{7} \mathrm{~cm} \mathrm{~s}^{-1}$. Under the above assumptions the set of the RMHD equations can be derived; they describe the evolution of magnetic and velocity fluctuations in terms of two distinct effects: (a) wave propagation in the longitudinal direction at the Alfvén velocity and (b) nonlinear couplings, which generate a turbulent cascade perpendicularly to $\boldsymbol{B}_{0}$. The model proposed by NMCV04 (hybrid shell model) includes both of these dynamical mechanisms, but nonlinear effects are described in a simplified way by using a shell technique (Boffetta et al. 1999): a Fourier expansion is carried out in the perpendicular directions, and the resulting spectral space is divided into concentric shells of exponentially increasing radius. In each shell, velocity and magnetic field fluctuations are represented by complex scalar quantities. Nonlinear effects are reproduced by quadratic terms representing the interactions between nearest and next nearest neighbor shells; the coefficients are chosen so as to conserve two-dimensional quadratic invariants: total energy, cross helicity, and squared magnetic potential. The equation of the hybrid shell model is written as

$$
\begin{aligned}
& \left(\frac{\partial}{\partial t}-\sigma \frac{\partial}{\partial s}\right) Z_{n}^{\sigma}(x, t)=-\chi k_{n}^{2} Z_{n}^{\sigma}(s, t) \\
& +i k_{n}\left(\frac{13}{24} Z_{n+2}^{\sigma} Z_{n+1}^{-\sigma}+\frac{11}{24} Z_{n+2}^{-\sigma} Z_{n+1}^{\sigma}-\frac{19}{48} Z_{n+1}^{\sigma} Z_{n-1}^{-\sigma}\right. \\
& \left.-\frac{11}{48} Z_{n+1}^{-\sigma} Z_{n-1}^{\sigma}+\frac{19}{96} Z_{n-1}^{\sigma} Z_{n-2}^{-\sigma}-\frac{13}{96} Z_{n-1}^{-\sigma} Z_{n-2}^{\sigma}\right)^{*}
\end{aligned}
$$

where $Z_{n}^{\sigma}(s, t)=v_{n \perp}(s, t)+\sigma b_{n \perp}(s, t)$ (with $n=0,1, \ldots, n_{\max }$ and $\sigma= \pm 1)$ are the Elsässer variables; $k_{n}=k_{0} 2^{n}$ is the transverse wavenumber, with $k_{0}=2 \pi\left(L / L_{\perp}\right) ; \chi=\lambda /\left(c_{\mathrm{A} 0} L\right)$, where the magnetic diffusivity $\lambda$ has been assumed equal to the transverse kinematic viscosity; and the asterisk means complex conjugate. Lengths are normalized to the loop length $L$, and time is normalized to the Alfvén transit time $t_{\mathrm{A}}=L / c_{\mathrm{A} 0}$; the velocity $v_{n \perp}$ and magnetic field $b_{n \perp}$ fluctuations are normalized to $c_{\mathrm{A} 0}$ and $\boldsymbol{B}_{0}$, respectively.

The shell technique allows us to describe the turbulence at high Reynolds/Lundquist numbers with a relatively small number of degrees of freedom. In particular, we used a number of shells, $n_{\max }=11$, with a very small dissipation coefficient, $\chi=$ $10^{-7}$. Since the longitudinal spatial dependence is retained, the hybrid shell model can describe effects of longitudinal resonance. Moreover, it is possible to implement boundary conditions to describe the effects of transverse motions at the loop bases. In particular, the system is excited through the boundary at $s=0$ by imposing a given velocity perturbation at large transverse scales, simulating photospheric motions. This boundary perturbation amounts to $\sim 10^{5} \mathrm{~cm} \mathrm{~s}^{-1}$, is Gaussian distributed, and has a correlation time $t_{c}=300 \mathrm{~s}$. At the other boundary $s=1$ total reflection conditions are imposed. Equation (5) is numerically solved using second-order finite difference schemes both in space and in time.

During the evolution, fluctuating energy enters or exits the driven boundary, so the total energy content in the loop fluctuates erratically in time. At the same time nonlinear effects transfer energy to smaller transverse scales, thus building a turbulence spectrum. Dissipation takes place mainly at the smallest scales. Occasionally, the velocity imposed at the lower boundary drives the loop close to one longitudinal resonance: then, the velocity fluctuations increase at the driven large-scale shells, enhancing the energy cascade process toward small dissipative scales. This process results in a spike of dissipated energy converted to heat. The dissipated power at time $t$ and position $s$ along the loop is calculated as

$$
H(s, t)=\frac{\chi}{2} \sum_{\sigma, n} k_{n}^{2}\left|Z_{n}^{\sigma}(s, t)\right|^{2}
$$

and is the heating input in the loop plasma model (eq. [3]). The hybrid shell model yields the energy distribution along the loop integrated in the transverse direction and therefore provides the heat input for the one-dimensional loop model. The power in the whole loop is

$$
W(t)=\int_{0}^{1} H(s, t) d s .
$$

The profile of $W(t)$ contains a sequence of spikes of different amplitudes and durations. The space and time profile of the heating function results from the interplay between the external driver (photospheric motions), the loop resonance, and the nonlinear turbulent cascade.

The heat spatial distribution is sampled every 0.1 Alfvén time. For an Alfvén speed of $2 \times 10^{8} \mathrm{~cm} \mathrm{~s}^{-1}$, one Alfvén transit time is $15 \mathrm{~s}$ (NMCV04). The numerical table yields the heat distribution per unit time and volume along the loop (sampled every $37.5 \mathrm{~km}$ ) and spans a total time of $307.5 \mathrm{ks}$, i.e., 3.56 days. We assume a circular cross section and an aspect ratio $d / L=0.2$, where $d$ is the cross section diameter; the cross section area is $A=2.83 \times$ $10^{17} \mathrm{~cm}^{2}$. Figure 1 shows a few selected segments of the evolution of the average loop heating rate $W(t) /(A L)$; they are essentially 

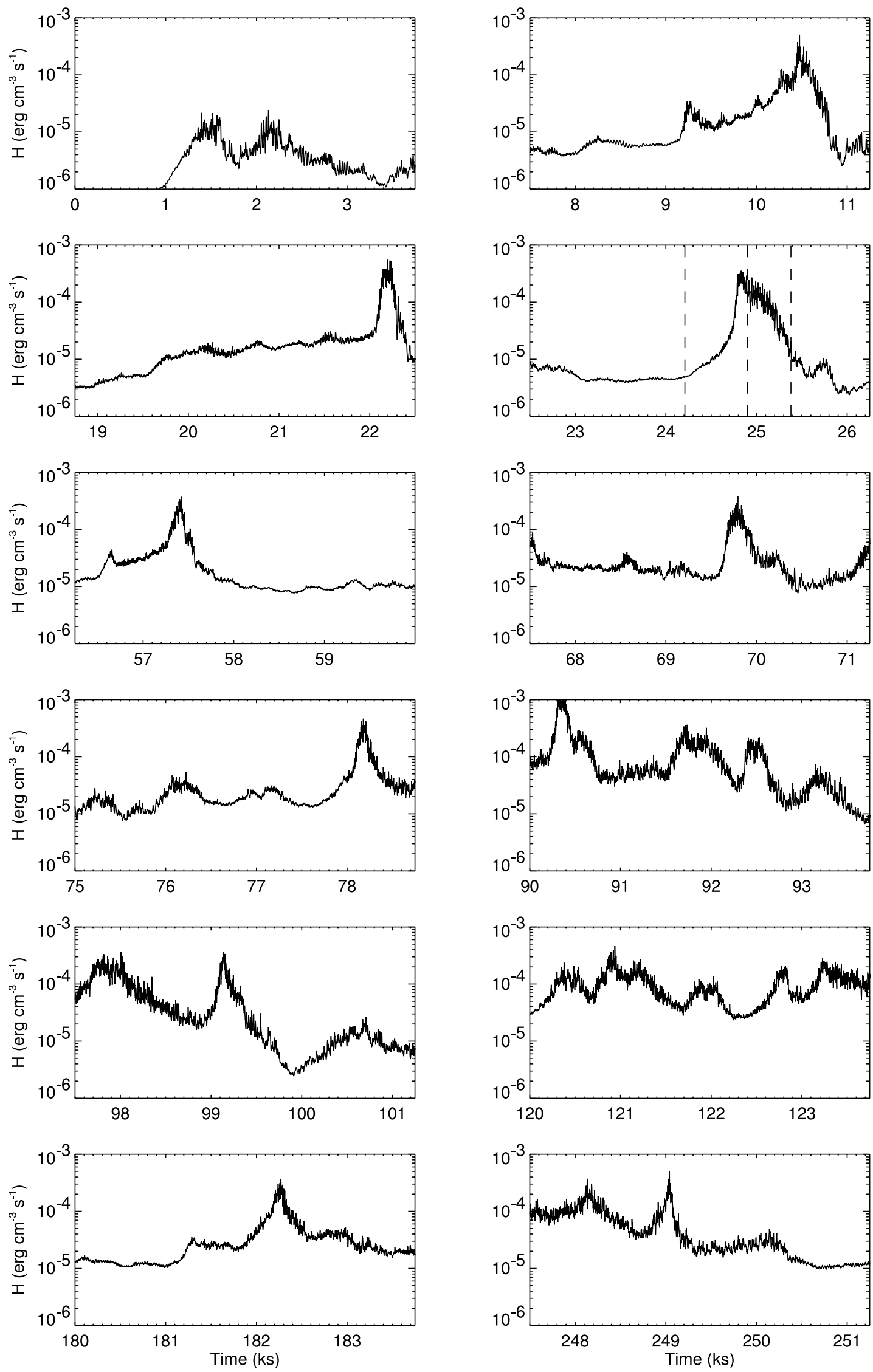

FIG. 1. Evolution of the average heating rate per unit volume released in the loop. The vertical dashed lines mark the times illustrated in detail in Fig. 2. 


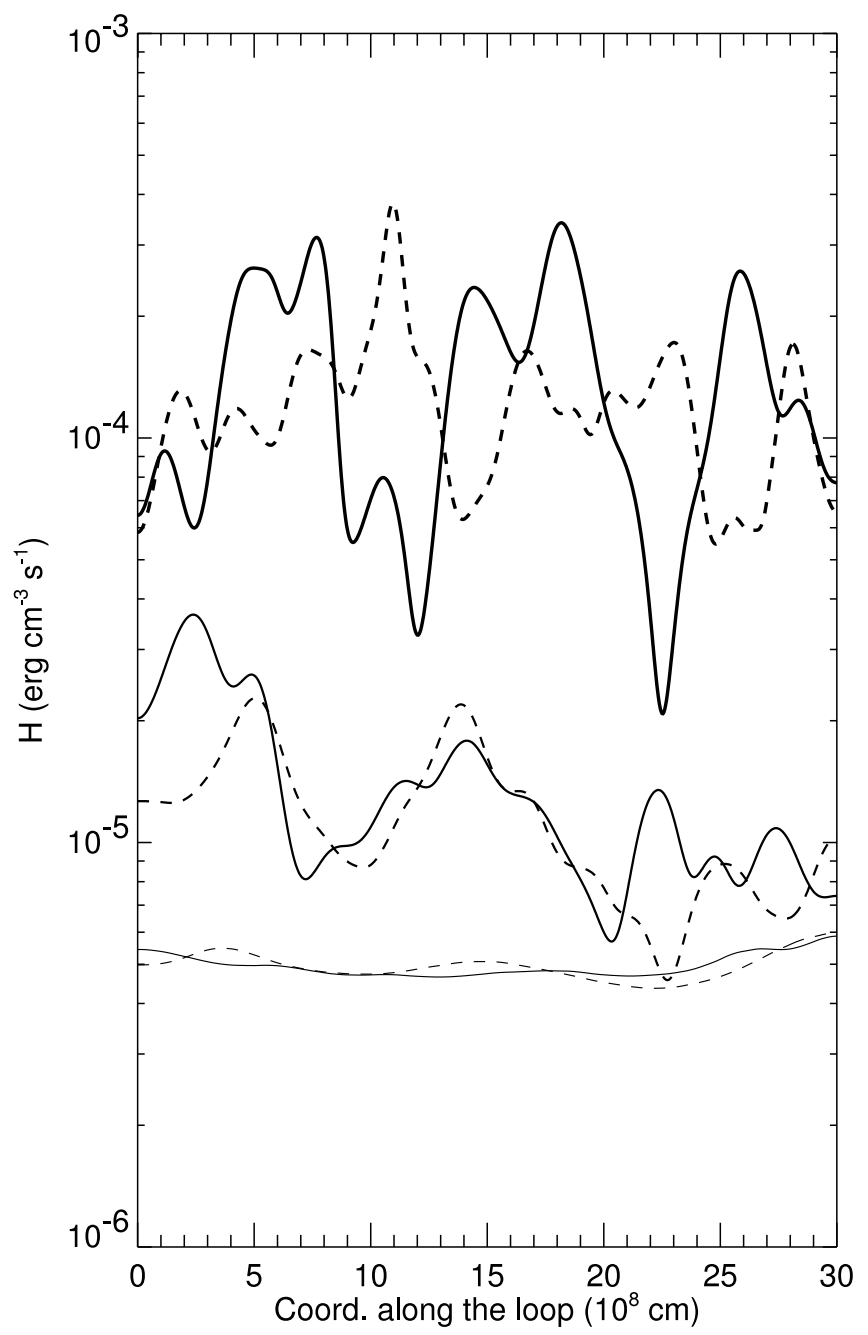

FIG. 2.- Spatial distributions of the heating rate per unit volume along the loop sampled during the fourth segment of Fig. 1 (second row, right) at the times marked. The dashed lines are the distributions after $1.5 \mathrm{~s}$ from the closest solid line.

zooms of the dissipation power shown in Figure 1 in NMCV04. The heating per unit volume is negligible in the first $1000 \mathrm{~s}$. After this (relatively short) transient, the heating is steadily above $10^{-6}$ ergs $\mathrm{cm}^{-3} \mathrm{~s}^{-1}$. The evolution of the average heating rate is highly irregular, with sharp pulses whose duration spans all timescales from a few s to a few ks. Some pulses resemble flares. Also, the pulse intensities are highly irregular. Most of them are entirely below $10^{-4}$ ergs $\mathrm{cm}^{-3} \mathrm{~s}^{-1}$. A few of them are higher (although most are below $10^{-3}$ ergs $\mathrm{cm}^{-3} \mathrm{~s}^{-1}$ ); in fact, 11 heating pulses reach values well above $3 \times 10^{-4} \mathrm{ergs} \mathrm{cm}^{-3} \mathrm{~s}^{-1}$ and occur around $10.5,22,25,57.5,69.5,78,90,99,121,182$, and $249 \mathrm{ks}$, as shown in Figure 1. The most intense pulse is the seventh one $(90 \mathrm{ks})$ and is higher than $10^{-3} \mathrm{ergs} \mathrm{cm} \mathrm{cm}^{-3} \mathrm{~s}^{-1}$. The high pulses are noticeably less frequent in the second half of the heating time interval: nine of them occur in the first $150 \mathrm{ks}$. Most of these pulses last $\sim 0.3-1 \mathrm{ks}$ and are rather peaked.

The heating rate per unit volume averaged over the whole heating duration is $\approx 3 \times 10^{-5} \mathrm{ergs} \mathrm{cm}^{-3} \mathrm{~s}^{-1}$. According to the loop scaling laws (Rosner et al. 1978), for the prescribed length this is the heating rate (per unit volume) of a loop at an equilibrium base pressure of $\approx 0.025 \mathrm{dyn}^{-2}$ and a maximum temperature of $\approx 5 \times 10^{5} \mathrm{~K}$.

Figure 2 shows distributions of the heating rate per unit volume along the loop sampled during the fourth segment in
Figure 1 (hereafter Ref1; from 22.5 to $27 \mathrm{ks}$ ). For each time, a couple of distributions are shown, one at $1.5 \mathrm{~s}$ from the other. The heating distribution is quite uniform for low heating. During the high-intensity phase of the heating, the distribution becomes less uniform, with large peaks propagating back and forth along the loop and extending over $\sim \frac{1}{5}$ of the loop.

\subsection{The Initial Conditions}

Since our scope is to investigate the structure, stability, and observable properties of the simulated loop both in time and on average, the initial conditions ought to be moderately important: we should start with an initially cool and empty loop, thereafter entirely governed by the new time-dependent heating. For technical reasons, our choice has been to set up this condition by allowing an initially hotter loop to relax to a much cooler condition. The initial loop is obtained from the model of Serio et al. (1981) with a uniform steady heating and a base pressure 0.03 dyn $\mathrm{cm}^{-2}$, corresponding to a loop maximum temperature of $\approx 5 \times 10^{5} \mathrm{~K}$, i.e., the expected average condition of the nanoflare-heated loop. In order to allow this loop to relax, we made a preliminary time-dependent simulation assuming zero coronal heating in the loop (but keeping the chromospheric heating on, to have stable footpoints). The simulation followed the loop evolution for $2000 \mathrm{~s}$, i.e., approximately 2.5 loop thermal decay times (Serio et al. 1991). At the end of the simulation, the loop maximum temperature decreased to $\sim 60,000 \mathrm{~K}$, and the pressure decreased to $\approx 1.5 \times 10^{-4} \mathrm{dyn}^{-2}$. A residual velocity field was present in the loop, with speeds not larger than $6 \mathrm{~km} \mathrm{~s}^{-1}$, an amply subsonic (Mach 0.2 ) value. We took this final status as the initial condition for the simulations with the nanoflare heating.

\section{RESULTS}

Our main purpose here is to explore how the dissipation rate described in NMCV04 can bring a loop to coronal conditions and maintain it. In this perspective we describe in detail the solution obtained in a segment containing a heat pulse of medium intensity, specifically Ref1 (between 22.5 and $26.3 \mathrm{ks}$ ) in Figure 1. We also discuss the segment including the highest heat pulse, i.e., the eighth segment (hereafter RefH). The solutions in the other segments do not differ much from those that we illustrate.

\subsection{Medium Pulse}

Figure 3 shows the evolution of the temperature, particle density, pressure, and velocity distributions along the loop obtained from the loop simulations for segment Ref1. The temperature is steadily below $0.2 \mathrm{MK}$ until the pulse at $t \approx 24.5 \mathrm{ks}$. Then it gradually increases due to the enhanced heating. Figure 3 clearly shows that the effects of the spatial heating structure (Fig. 2) are smoothed by the efficient thermal conduction. The pulse also drives plasma evaporation from the chromosphere, visible in the density, pressure, and velocity distributions (the negative velocity peaks indicate plasma moving upward from the far footpoint). The density distributions show more significant fluctuations traveling along the loop.

For more quantitative information, Figure 4 shows selected distributions of temperature, particle density, velocity, and pressure along the loop around the times marked in Figure 2. Each column of the figure shows the distributions along the loop at the exact time, as well as $100 \mathrm{~s}$ before and after this time. In the low heating state (Fig. 4, left column), the temperature is steadily between 0.2 and $0.3 \mathrm{MK}$ along most of the loop, with a profile 

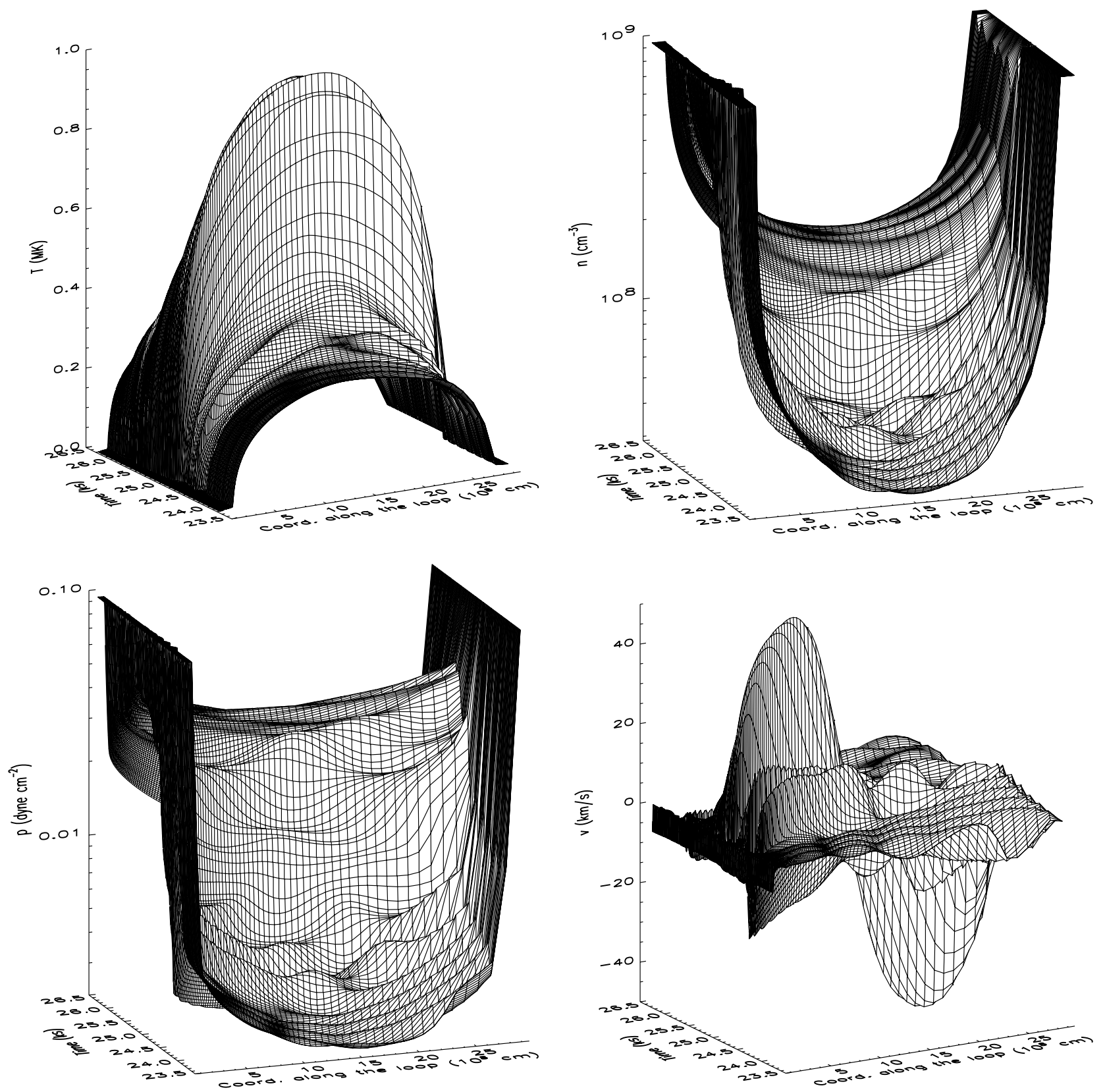

FIG. 3.-Evolution of the distributions of temperature, particle density, velocity, and pressure along the loop during segment Ref1 (the fourth segment of Fig. 1, second row, right).

very similar to that of a static loop. Also, the density does not change much along the loop and is always below $10^{8} \mathrm{~cm}^{-3}$ in most of the loop. The distribution of plasma velocity shows fluctuations with amplitude $\sim 10 \mathrm{~km} \mathrm{~s}^{-1}$ propagating back and forth along the loop. During the heat pulse, the temperature increases to about $1 \mathrm{MK}$ (in $\sim 100 \mathrm{~s}$ ). The distribution at the time of the temperature maximum appears to be more peaked than in the cool state, and the position of the maximum slightly oscillates around the loop apex. At later times $(t>25 \mathrm{ks})$, the temperature slowly decreases, and its distribution flattens (Fig. 4, first row, right). Asymmetric fronts of plasma evaporation develop as the heating increases (Fig. 4, second row, center) and the density starts to increase. The density continues to increase even after the temperature maximum (Fig. 4, second row, right), staying above $2 \times 10^{8} \mathrm{~cm}^{-3}$ for a long time. During the heat pulse, the plasma evaporation fronts are also clearly visible in the veloc- ity profiles: two similar strong fronts rise from both footpoints after $t=24.8 \mathrm{ks}$, reaching a speed of about $50 \mathrm{~km} \mathrm{~s}^{-1}$ at intermediate positions along the loop. Then the plasma becomes noticeably less dynamic. During the heating decay, the loop slowly returns to a cool average state around $0.4 \mathrm{MK}$. The plasma velocity continues to decrease until the plasma becomes practically static, around $t=25.5 \mathrm{ks}$. Then the velocity distribution gets inverted: plasma begins to drain along the loop at a very low speed (lower than $10 \mathrm{~km} \mathrm{~s}^{-1}$ ). The pressure distribution along the loop is quite stable in the cool state. When the heating increases, the pressure increases as well (together with the temperature and the density). The pressure distribution then settles to a very flat distribution during the pulse decay at about $0.04 \mathrm{dyn} \mathrm{cm}^{-2}$.

Figure 5 shows the evolution of the loop maximum temperature, the loop minimum density and pressure, and the maximum velocity. The first three quantities are typical of the upper region 

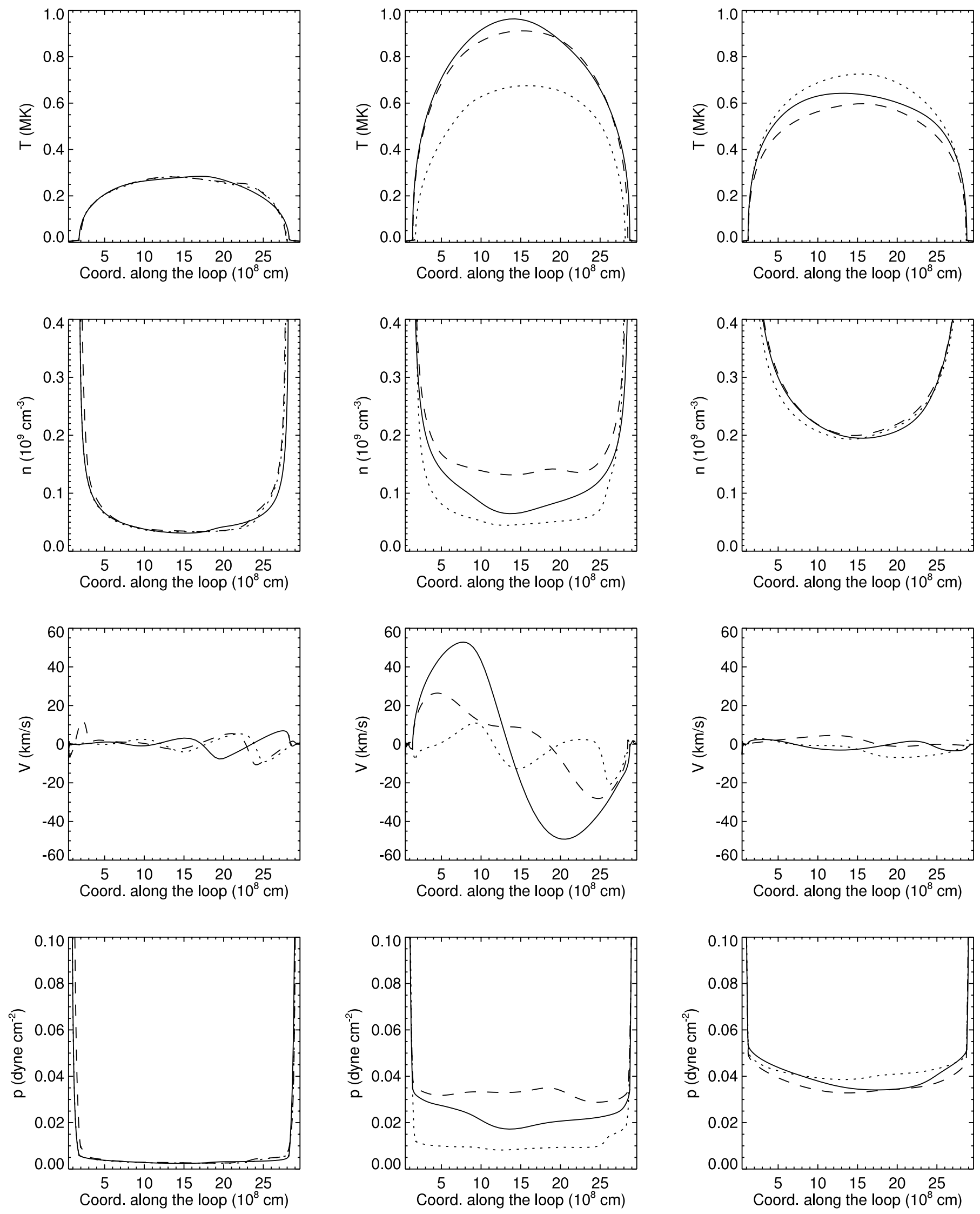

FIG. 4.-Distributions of temperature, particle density, velocity, and pressure along the loop sampled during segment Ref1 (the fourth segment in Fig. 1, second row, right) at the three times marked in Fig. 2 (one for each column). We show the distributions at these times (solid lines), as well as $100 \mathrm{~s}$ before (dotted lines) and after (dashed lines). 

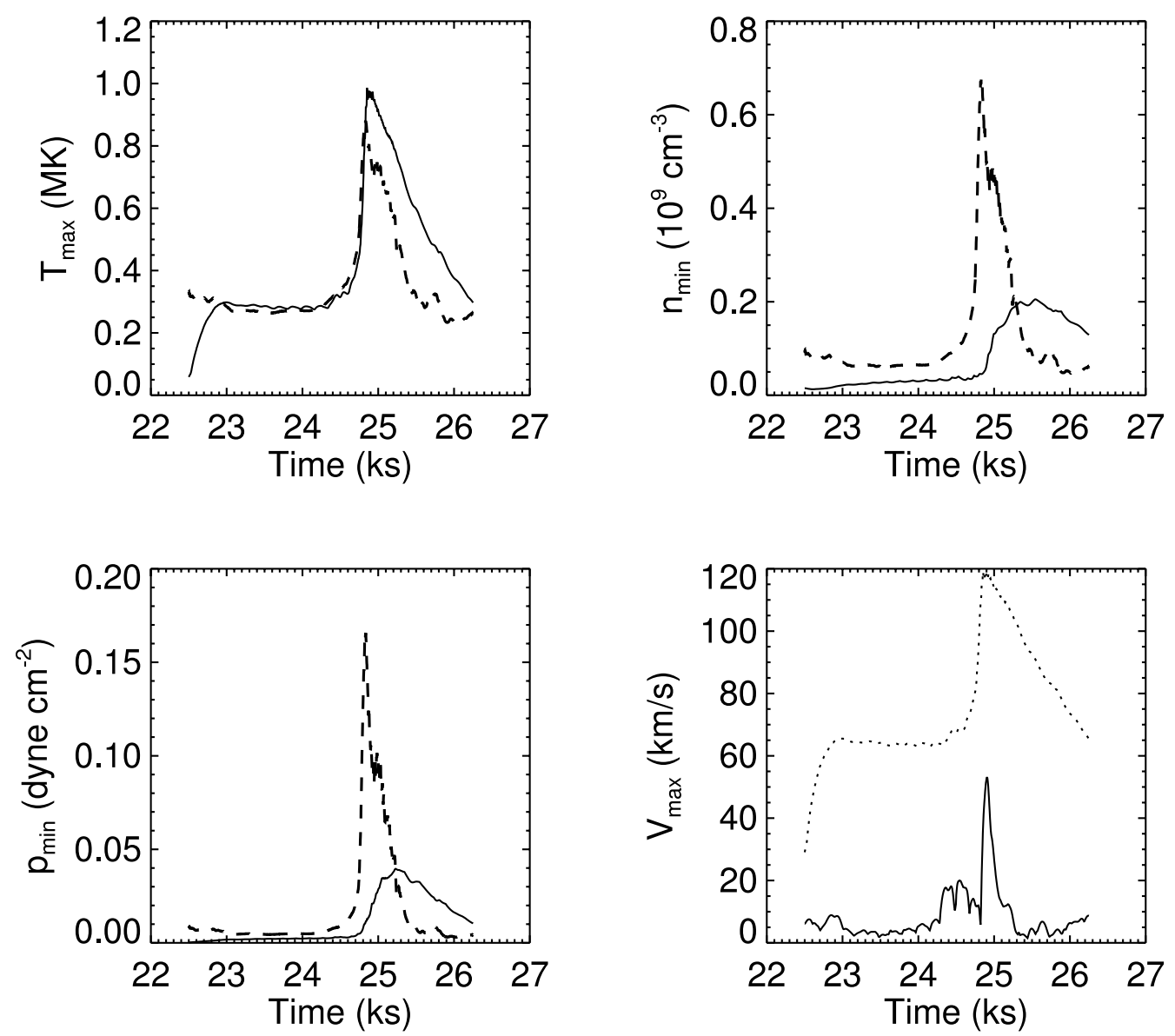

FIG. 5.-Evolution of the loop maximum temperature, minimum density, minimum pressure, and maximum velocity along the loop during segment Ref1. The dashed lines indicate the loci of the equilibrium conditions of the loop according to the loop scaling laws, corresponding to the heating evolution in Fig. 1. In the velocity plot, the dotted line is the sound speed at the loop maximum temperature.

of the loop, close to the apex, the last midway between the apex and the footpoint of the loop. The evolution of the maximum loop temperature is globally similar to that of the average heating (Fig. 1), but much less noisy. Consequently, it is also similar to the evolution of the maximum temperature expected from the evolution of the average loop heating through the loop scaling laws (Rosner et al. 1978). The former temperature is slightly higher $(\sim 10 \%)$ and decays more slowly than the latter one. The peak temperature is different because scaling laws assume a constant and uniform heating, while the actual heating function in the simulation is variable and nonuniform along the loop. The slower decay is due to the fact that the plasma response to the heating decrease is not instantaneous, and the cooling processes have their own characteristic times. The density enhancement due to the heat pulse of this segment is significantly delayed ( $\sim 300 \mathrm{~s}$ ) with respect to the temperature increase, as is typical of loop plasma evaporation. For comparison, Figure 5 shows the equilibrium loop density values as expected from the loop scaling laws. The comparison clearly shows the delay mentioned above but also emphasizes that during the pulse rise the loop is significantly underdense yet becomes overdense in the later decay phase. This is expected in dynamically heated loops; while the heating is on, the loop is filling with plasma and therefore below the density equilibrium conditions. When the heating stops, the loop cools down, but the plasma drains even more slowly. The maximum pressure has an evolution between that of the density and of the temperature and explains why the plasma dynamics is time shifted with respect to the plasma thermal evolution. Figure 5 shows that the plasma velocity is constantly below $20 \mathrm{~km} \mathrm{~s}^{-1}$ except during the heat pulse, when it grows to about $50 \mathrm{~km} \mathrm{~s}^{-1}$. These values are quite subsonic.

From the output results of the hydrodynamic simulations, i.e., distributions of temperature, density, and velocity along the loop sampled at regular time intervals, it is possible to compute the UV and X-ray emission from the confined plasma. Figure 6 shows the emission along the loop in three representative X-ray/ $\mathrm{UV}$ lines, i.e., $\mathrm{Ca} \times \lambda 558, \mathrm{Mg} \mathrm{IX} \lambda 368$, and $\mathrm{Mg} \times \lambda 625$, peaking at $\log T=5.9,6.0$, and 6.1, respectively, at the same times as the distributions shown in the left and middle columns of Figure 4. Since the line emission is sensitive both to the temperature and to the square of the density, the emission distributions are less uniform and fluctuate more. This may be a distinctive signature of this model in loop observations. In these lines the loop is visible for a limited time during this segment. In the hottest line ( $\mathrm{Mg} \times 2625)$ it decays very rapidly.

\subsection{High Pulse}

In the course of the whole sequence of heating evolution, the most intense heat pulse, Ref H, occurs a little after time $t=90 \mathrm{ks}$ (Fig. 1, fourth row, right column). Figure 7 shows the evolution of the loop maximum temperature, the loop minimum density and pressure, and the maximum velocity, to be compared with the evolution obtained in segment Ref1 (Fig. 5). The loop maximum temperature reaches $1.5 \mathrm{MK}$ around time $t=90.5 \mathrm{ks}$. 

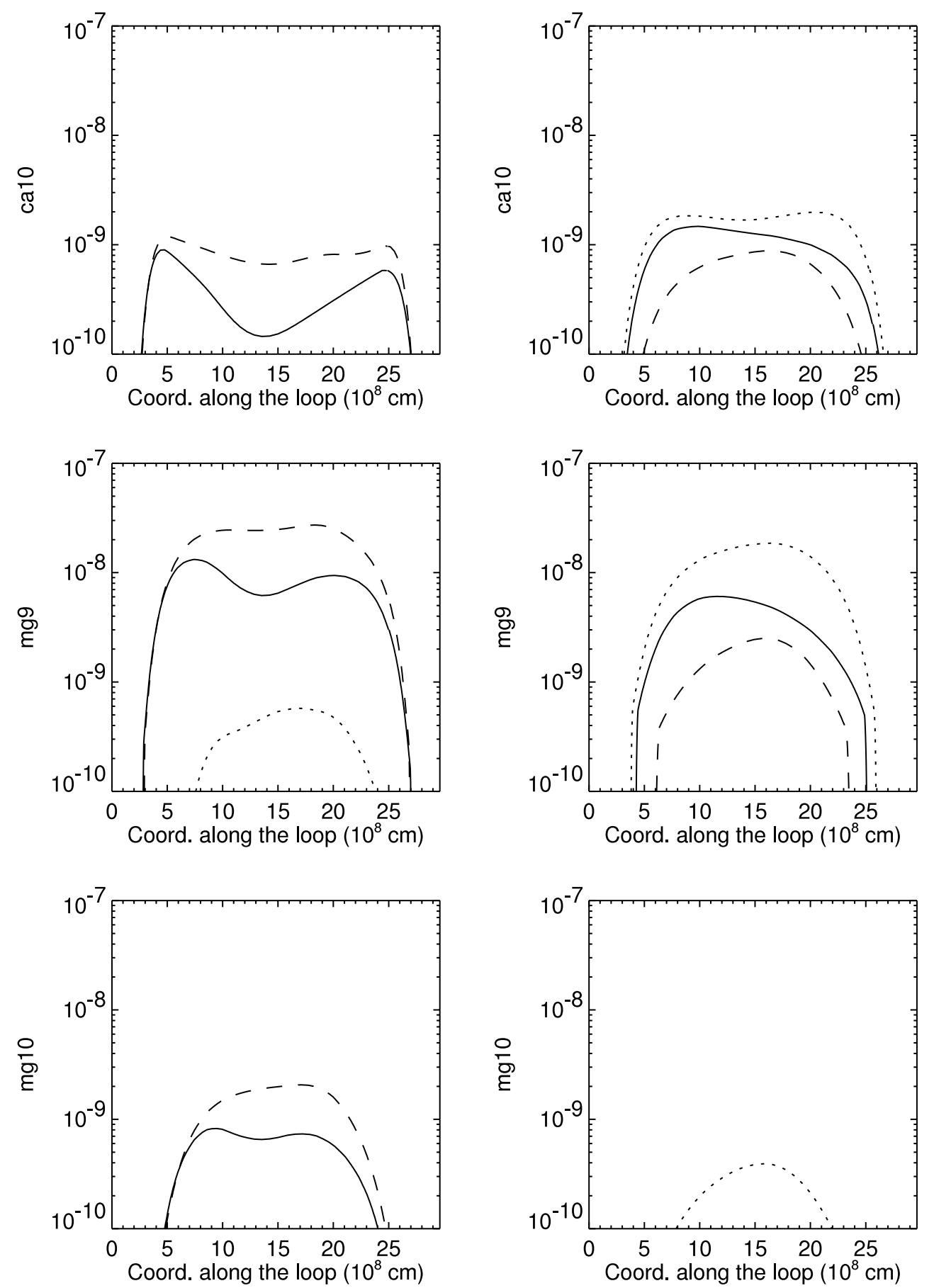

FIG. 6.-Emission distributions (in ergs $\mathrm{cm}^{-3} \mathrm{~s}^{-1}$ ) along the loop in three relevant X-ray/UV lines (Ca x $\lambda 558, \mathrm{Mg} \mathrm{IX} \lambda 368$, and $\left.\mathrm{Mg} \mathrm{x} \lambda 625\right)$ during segment Ref1 at the same times as the left and middle columns of Fig. 4. For the chosen loop parameters, $10^{-10} \mathrm{ergs}^{-3} \mathrm{~s}^{-1}$ is a reasonable threshold for detection. 

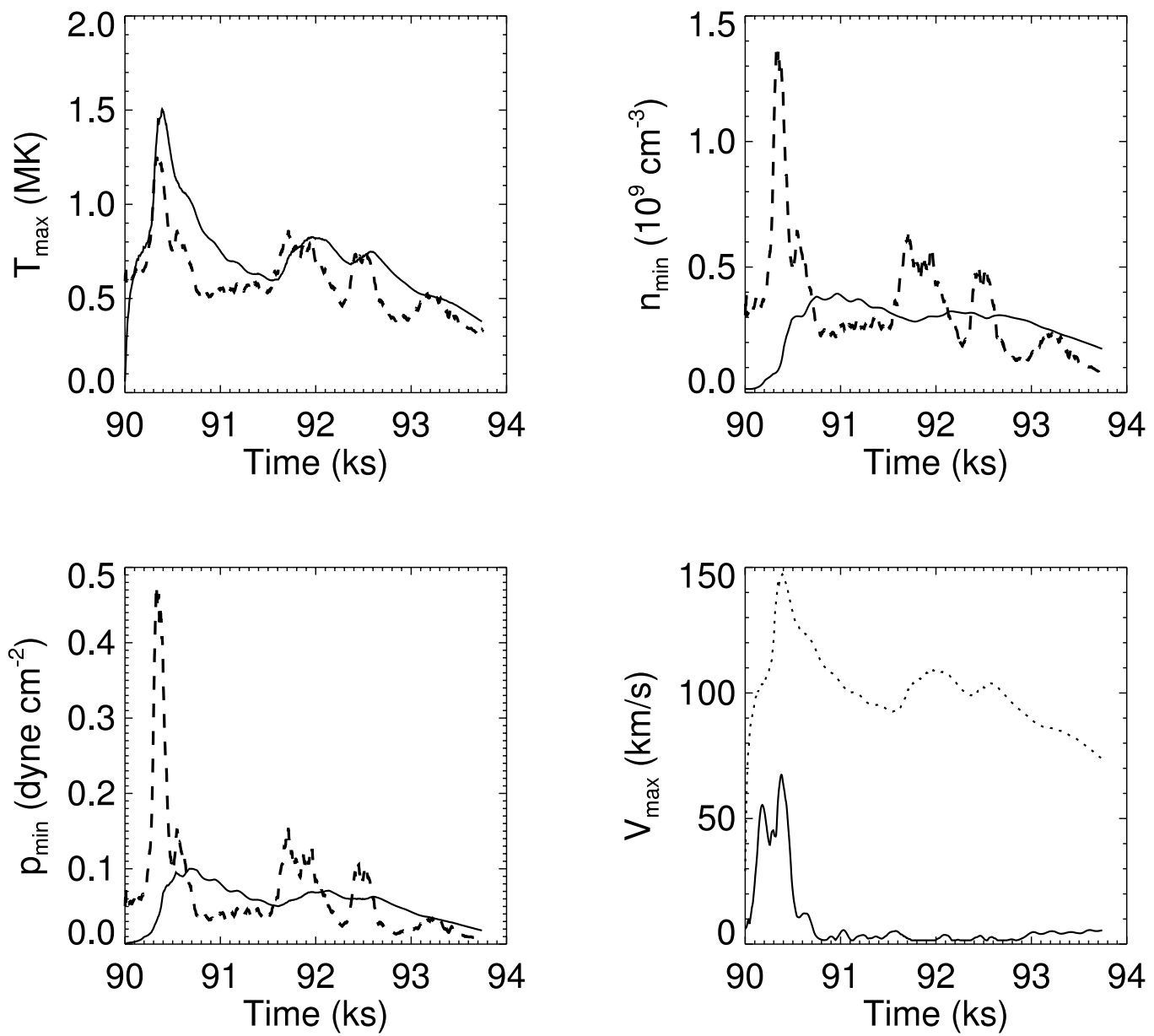

FIG. 7.-Evolution of the loop maximum temperature, minimum density, minimum pressure, and maximum velocity along the loop during segment Ref H. The dashed and dotted lines are the same as in Fig. 5.

Then it decays below $1 \mathrm{MK}$, but stays above $0.5 \mathrm{MK}$ for the rest of the segment because of the occurrence of other minor heat pulses. The density at the apex reaches about $4 \times 10^{8} \mathrm{~cm}^{-3}$, and the pressure reaches 0.1 dyn $\mathrm{cm}^{-2}$ around time $t=91 \mathrm{ks}$, about $500 \mathrm{~s}$ later than the temperature peak. The velocity gets above $60 \mathrm{~km} \mathrm{~s}^{-1}$, always amply subsonic.

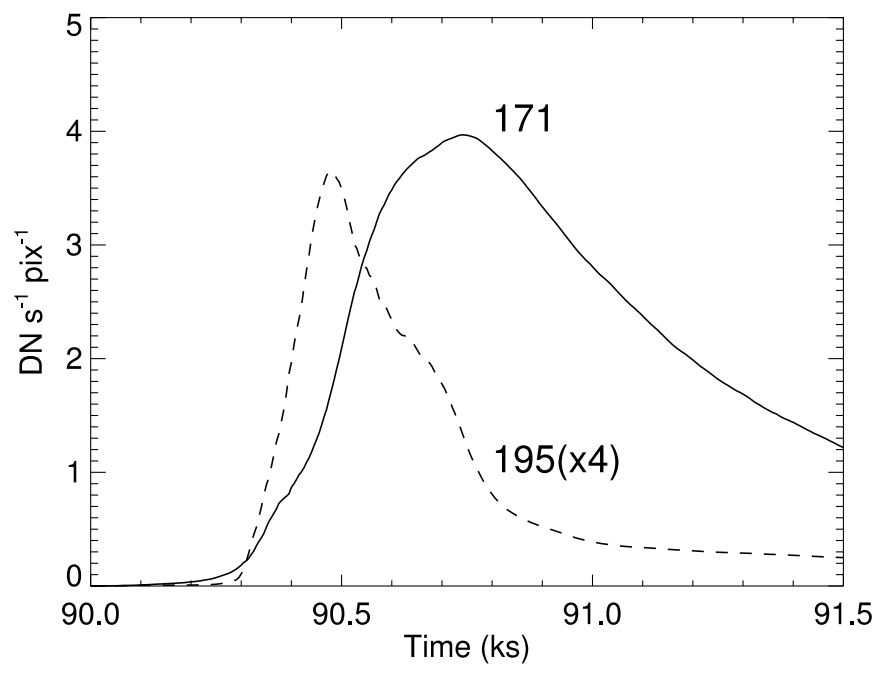

Fig. 8. - Light curves integrated along the whole loop during segment Ref $\mathrm{H}$ in the $171 \AA$ (solid line) and in the $195 \AA$ (dashed line) filter bands of the TRACE telescope. The $195 \AA$ emission is multiplied by 4 .
Figure 8 shows the light curves integrated along the whole loop during segment RefH in the 171 and $195 \AA$ filter bands of the Transition Region and Coronal Explorer (TRACE; Handy et al. 1999). The light curve in the 171 Å filter band resembles the evolution of the heat pulses (although much smoother). In the $195 \AA$ filter band, only the first pulse is significant, and only in its initial phase is the emission significant, giving the impression of an anticipated evolution. This evolution resembles more closely the evolution of the maximum temperature shown in Figure 7.

\section{DISCUSSION AND CONCLUSIONS}

This work is devoted to exploring the effect of nanoflares due to the magnetic energy dissipation through MHD turbulence on the dynamic and thermal evolution of the plasma in a coronal loop. The parameters considered in NMCV04, i.e., an Alfvén speed of $2000 \mathrm{~km} \mathrm{~s}^{-1}$ corresponding to a magnetic field of about $10 \mathrm{G}$ in corona, lead to a loop with a typical maximum temperature of $5 \times 10^{5} \mathrm{~K}$. Since coronal loops are typically observed at higher temperatures, $\geq 1 \mathrm{MK}$, here we focus on the effects produced by the most intense heat pulses predicted in NMCV04. We compute in detail the hydrodynamics and thermodynamics of the loop plasma during the pulses and analyze the results.

Although the spatial distribution of the heating has significant fluctuations traveling along the loop and also rapid fluctuations in time, we find that the plasma is not so fast to react and smooths out the fluctuations both in space and in time. We find that, under the effect of a medium heat pulse, the loop plasma reaches $T \sim 1$ MK and density $\sim 0.2 \times 10^{9} \mathrm{~cm}^{-3}$. The efficient thermal 
conduction makes the plasma respond promptly to the heating deposition but also smooths the heating fluctuations. The plasma rapidly reaches the equilibrium temperature (according to the loop scaling laws) and then cools following the decay of the heat pulse. The same evolution occurs for a higher heat pulse, which produces a higher peak temperature of $1.5 \mathrm{MK}$ and a higher density of $0.5 \times 10^{9} \mathrm{~cm}^{-3}$. The density (and pressure) of the plasma shows more significant fluctuations traveling along the loop but globally responds on longer timescales. The heat pulses do not last long enough to let the plasma reach the thermo-/hydrostatic equilibrium: the plasma is underdense during the heat pulse and overdense after the pulse with respect to thermal equilibrium. This density evolution is a consequence of the impulsive heating (Winebarger et al. 2003a; Warren et al. 2003). The speed of the plasma driven by the heat pulse is relatively small, largely subsonic, and speeds of a few tens of $\mathrm{km} \mathrm{s}^{-1}$ occur only for very few minutes. The emission distribution in relevant spectral lines may be relatively more sensitive to fluctuations due to the turbulent heating and may be used to diagnose this model. For the highest heat pulse, our model also predicts the light curves in two relevant TRACE filter bands to be "out of phase" from each other. This phase difference is in qualitative agreement with observations (Winebarger et al. 2003b) but is also predicted by other loop models (Warren et al. 2003).

The heating model used here has very few free parameters (essentially the magnetic field strength and the loop length) and depends on basic physical effects. The shell model does not yield a detailed description of turbulence and cannot reproduce the energy distribution in the direction transverse to the magnetic field. However, it should be adequate to describe the behavior of the loop integrated in the transverse direction and the detailed energy dissipation along the loop, matching the scope of the Palermo-Harvard loop model.

A series of questions is opened by this work. First, characteristic features of the proposed heating are the disturbances traveling along the loop. We have shown that observations in single spectral lines may be sensitive to disturbances in the loop, but detecting such effects may not be trivial with present-day instruments. Also, one may wonder about the effect of changing the magnetic field strength: can a stronger field lead to hotter active region loops or even major flares? Even if the heating function can be modified with a simple scaling, this possibility still requires additional detailed loop modeling, since the loop plasma evolves nonlinearly under the effect of the heating, coupled with the dynamics and the cooling processes.

As a further issue to investigate, we note that the heating function is modified by the local plasma conditions, e.g., the density stratification and its time variation (i.e., the Alfvén speed depends on the density). Self-consistently including feedback of the loop plasma conditions on the energy dissipation may easily modify some characteristics of the heating function, such as the pulse duration, and thus influence the results. Tackling this question requires the coupling of the hybrid MHD turbulence model with the loop time-dependent hydrodynamic model, a task planned for future work.

This first work paves the road to future works along several lines, such as the time decomposition analysis of results, the coupling of the heating and loop models, and comparison with observations, encompassing the selection (or acquisition) and analysis of observations with long and regularly sampled image sequences.

F. R. and G. P. acknowledge support for this work from Ministero dell'Istruzione, Università e Ricerca. G. N., F. M., and P. V. acknowledge partial support by MIUR (Ministero dell Istruzione, dell'Università e della Ricerca) through a National Project Fund (COFIN 2002) and by the European Community within the Research Training Network Turbulence in Space Plasmas, Theory, Observation and Simulation. RMHD numerical calculations were performed in the framework of HPCC (Center for High Performance Computing) of the University of Calabria.
Aschwanden, M. J. 1999, Sol. Phys., 190, 233

Aschwanden, M. J., Nightingale, R. W., Tarbell, T. D., \& Wolfson, C. J. 2000a, ApJ, 535, 1027

Aschwanden, M. J., Tarbell, T. D., Nightingale, R. W., Schrijver, C. J., Title, A., Kankelborg, C., Martens, P., \& Warren, H. P. 2000b, ApJ, 535, 1047

Betta, R., Peres, G., Reale, R., \& Serio, S. 1997, A\&AS, 122, 585

Boffetta, G., Carbone, V., Giuliani, P., Veltri, P., \& Vulpiani, A. 1999, Phys. Rev. Lett., 83, 4662

Buchlin, E., Aletti, V., Galtier, S., Velli, M., Einaudi, G., \& Vial, J.-C. 2003, A\&A, 406, 1061

Carbone, V., \& Veltri, P. 1990, Geophys. Astrophys. Fluid Dyn., 52, 153

Cargill, P. J. 1993, Sol. Phys., 147, 263

Cargill, P. J., \& Klimchuk, J. A. 1997, ApJ, 478, 799 2004, ApJ, 605, 911

Crosby, N. B., Aschwanden, M. J., \& Dennis, B. R. 1993, Sol. Phys., 143, 275

Datlowe, D. W., Elcan, M. J., \& Hudson, H. S. 1974, Sol. Phys., 39, 155

Dennis, B. R. 1985, Sol. Phys., 100, 465

Dmitruk, P., \& Gómez, D. O. 1997, ApJ, 484, L83 1998, ApJ, 505, 974 1999, ApJ, 527, L63

Einaudi, G., Velli, M., Politano, H., \& Pouquet, A. 1996, ApJ, 457, L113

Handy, B. N., et al. 1999, Sol. Phys., 187, 229

Hendrix, D. L., \& Van Hoven, G. 1996, ApJ, 467, 887

Judge, P. G., Hansteen, V., Wikstol, O., Wilhelm, K., Schuehle, U., \& Moran, T. 1998, ApJ, 502, 981

Katsukawa, Y., \& Tsuneta, S. 2001, ApJ, 557, 343

Kopp, R. A., \& Poletto, G. 1993, ApJ, 418, 496

Krucker, S., \& Benz, A. O. 1998, ApJ, 501, L213

Lin, R. P., Schwartz, R. A., Kane, S. R., Pelling, R. M., \& Hurley, K. C. 1984, ApJ, 283, 421

\section{REFERENCES}

Mitra-Kraev, U., \& Benz, A. O. 2001, A\&A, 373, 318

Müller, D. A. N., Peter, H., \& Hansteen, V. H. 2004, A\&A, 424, 289

Nigro, G., Malara, F., Carbone, V., \& Veltri, P. 2004, Phys. Rev. Lett., 92, 194501 (NMCV04)

Parker, E. N. 1988, ApJ, 330, 474

Parnell, C. E., \& Jupp, P. E. 2000, ApJ, 529, 554

Peres, G., Reale, F., \& Serio, S. 1993, in Physics of Solar and Stellar Coronae, ed. J. L. Linsky \& S. Serio (Dordrecht: Kluwer), 151

Peres, G., Reale, F., Serio, S., Pallavicini, R. 1987, ApJ, 312, 895

Peres, G., Rosner, R., Serio, S., \& Vaiana, G. S. 1982, ApJ, 252, 791

Reale, F., Peres, G., Serio, S., Betta, R. M., DeLuca, E. E., \& Golub, L. 2000, ApJ, 535, 423

Rosner, R., Tucker, W., \& Vaiana, G. 1978, ApJ, 220, 643

Saba, J. L. R., \& Strong, K. T. 1991, ApJ, 375, 789

Serio, S., Peres, G., Vaiana, G. S., Golub, L., \& Rosner, R. 1981, ApJ, 243, 288

Serio, S., Reale, F., Jakimiec, J., Sylwester, B., \& Sylwester, J. 1991, A\&A, 241,197

Shimizu, T. 1995, PASJ, 47, 251

Shimizu, T., \& Tsuneta, S. 1997, ApJ, 486, 1045

Spadaro, D., Lanza, A. F., Lanzafame, A. C., Karpen, J. T., Antiochos, S. K.,

Klimchuk, J. A., \& MacNeice, P. J. 2003, ApJ, 582, 486

Spitzer, L. 1962, Physics of Fully Ionized Gases (New York: Wiley)

Strauss, H. R. 1976, Phys. Fluids, 19, 134

Testa, P., Peres, F., \& Reale, F. 2005, ApJ, 622, 695

Warren, H. P., Winebarger, A. R., \& Hamilton, P. S. 2002, ApJ, 579, L41 Warren, H. P., Winebarger, A. R., \& Mariska, J. T. 2003, ApJ, 593, 1174 Winebarger, A. R., Warren, H. P., \& Mariska, J. T. 2003a, ApJ, 587, 439 Winebarger, A. R., Warren, H. P., \& Seaton, D. B. 2003b, ApJ, 593, 1164 Zank, G. P., \& Matthaeus, W. H. 1992, J. Plasma Phys., 48, 1 